\title{
Fibroma of tendon sheath located within the ankle joint capsule
}

\author{
Riccardo Ciatti · Pier Paolo Mariani
}

Received: 29 July 2008/Accepted: 11 July 2009/Published online: 31 July 2009

(C) Springer-Verlag 2009

\begin{abstract}
We report a very rare case of fibroma of the tendon sheath arising from the anteromedial ankle joint capsule, with no apparent connection to any tendon in the area, found in a 58-year-old patient complaining of progressive local swelling. This uncommon tumor has its usual localization in tendon sheaths, is extremely rare in joint capsules, and has never been described in this location previously. MRI showed nonuniform low signal intensity in T1- and T2-weighted images and high intensity in STIR images. The mass was completely excised by open surgery. Histopathological analysis later confirmed the diagnosis of a fibroma of the tendon sheath.
\end{abstract}

Keywords Tumor Tendon sheath · Fibroma .

Ankle joint

\section{Introduction}

Fibroma of the tendon sheath is a rare tumor described as a benign lesion or a tumor-like reactive lesion arising from the synovium of tendon sheath [14]. It has been reported mainly in finger and hand tendons as a benign, slowly growing nodule that arises from a synovial sheath $[2,6,15,16,18]$.

\footnotetext{
R. Ciatti

Casa di Cura "Villa Stuart", Via Trionfale 5952,

00135 Rome, Italy

P. P. Mariani

Istituto Universitario di Scienze Motorie,

P.le Lauro de Bosis 15, 00194 Rome, Italy

R. Ciatti $(\bowtie)$

Via Pellegrino Matteucci 134, 00154 Rome, Italy

e-mail: riccardo.ciatti@libero.it
}

Involvement as a mass adjoining the synovial membrane of a joint capsule is extremely rare, and to our knowledge only seven cases have been described, mainly in the knee joint (four cases) [7, 8, 10, 12-14, 18]. We wish to emphasize its unusual location in the case described here-in the ankle-a location for a fibroma of the tendon sheath that has never been described in the English literature before.

\section{Case report}

This case concerns a 58-year-old man presenting a 24-month history of progressive localized swelling in the anteromedial aspect of his right ankle joint, with no recollection of associated trauma. The mass was slow-growing up to three months before coming to our attention, when it began to grow rapidly. Physical examination revealed an approximately 5-cm-diameter ovoid mass over the anteromedial aspect of the right ankle joint; the range of motion for dorsiflexion was progressively reduced to $10^{\circ}$, and was slightly painful beyond this range. Some discomfort was elicited at pressure over the mass. No neurologic or vascular compression symptoms were observed. He had no diffuse joint effusion nor any other particular findings on other physical examinations. Routine laboratory data were normal. The mass was noted to be mobile within its surrounding layers.

Plain X-rays of the right ankle joint were normal, while an MRI scan of the same region showed a soft tissue mass $5.5 \times 3.4 \times 2.6 \mathrm{~cm}$ in size arising from the anteromedial joint capsule. The mass had a nonuniform low intensity in T1- and T2-weighted scans, with focal septated areas exhibiting more intense signals (Fig. 1a). In STIR scans, the mass had a more uniform high intensity (Fig. 1b).

The patient underwent an excision of the mass by anteromedial incision carried out under peripheral 
Fig. 1 a Sagittal T1-weighted MRI. An anteromedial ankle joint mass (arrow) with a nonuniform low intensity, along with focal septated areas exhibiting more intense signals. b Sagittal STIR MRI scan. The mass (arrow) shows a more uniform high intensity
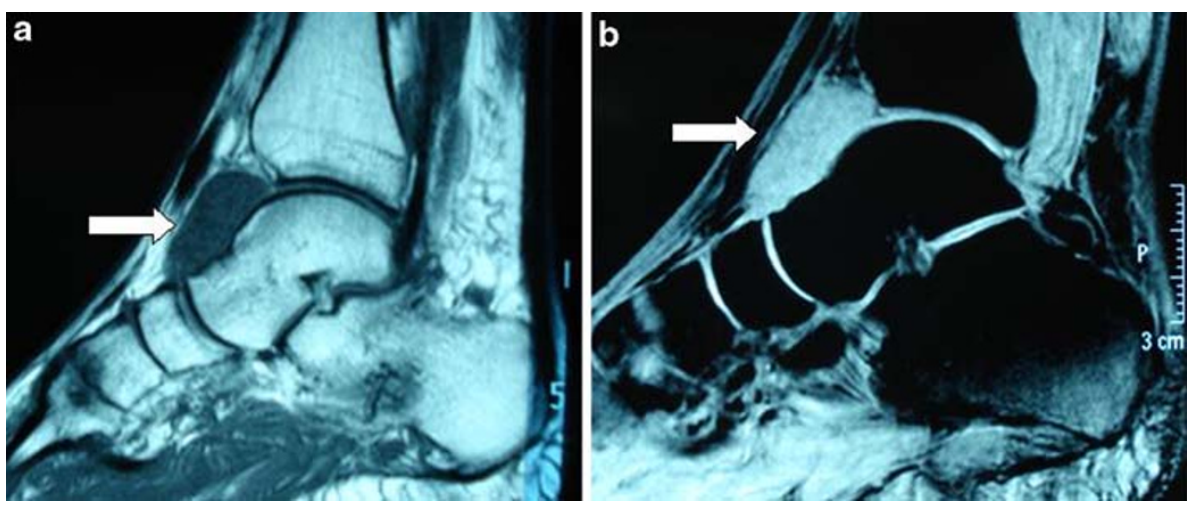

Fig. 2 a Anteromedial incision showing a distinct mass dislocating the lesser saphenous vein posteromedially.

b Removal of the mass: the multilobular aspect is seen. c At section, the mass appears to be gray-pearly white, multilobular and solid
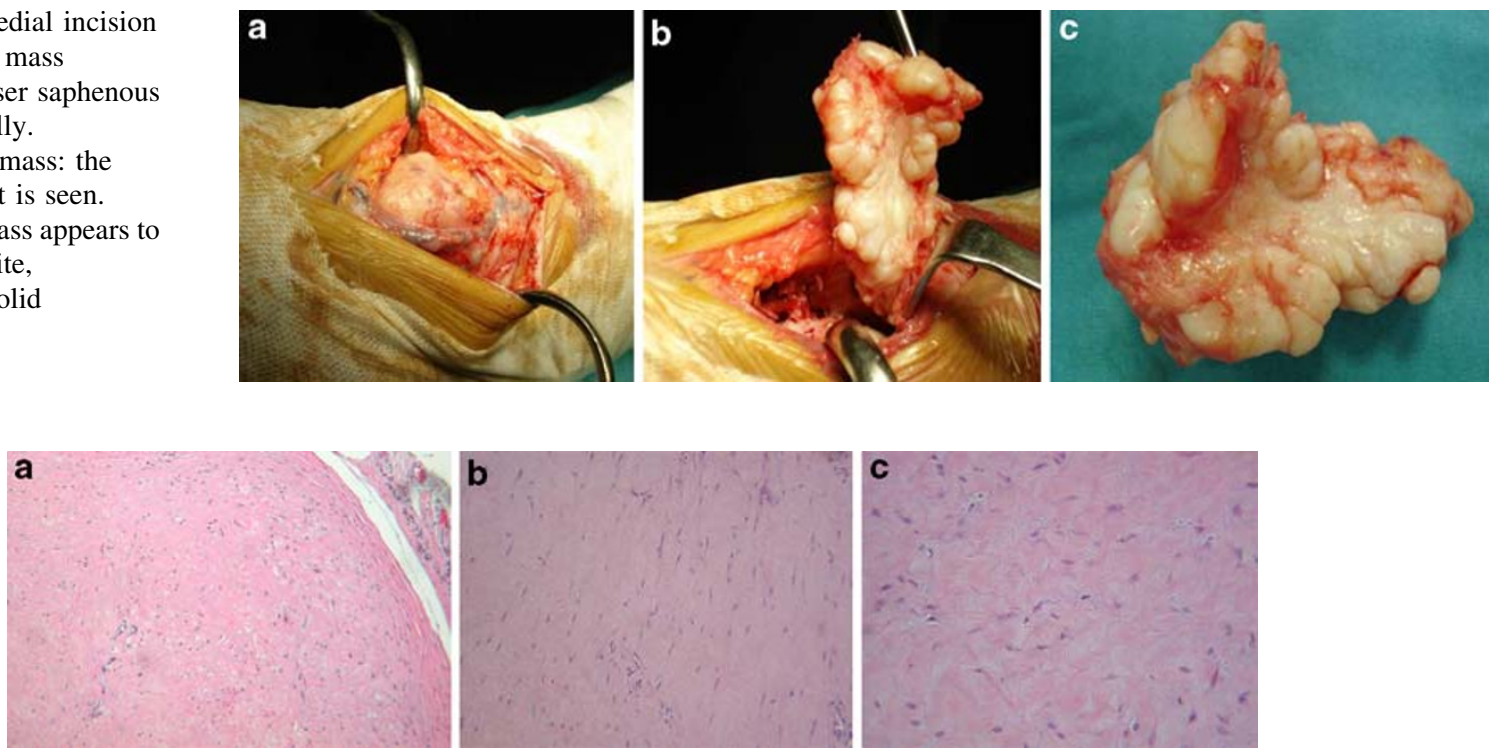

Fig. 3 a $H \& E \times 10$, the lesion appears to be circumscribed and surrounding tissues (right) are compressed by its growth. Cellularity is poor. b $\mathrm{H} \& \mathrm{E} \times 25$, tumor cells appear to be spindle-shaped and

anesthesia (Fig. 2a). On exploration, the mass was adherent to the lesser saphenous vein, which was isolated and medially mobilized. No clear capsule nor cleavage layer was found with the joint capsule, and no vascular peduncle was found either, so that part of the anteromedial ankle joint capsule had to be removed with the mass. No localized infiltration into surrounding tissues was macroscopically observed. Upon inspection, the mass appeared to be a fibrous structure with a nonrubbery, hard consistency, and was graypearly white, multilobular and solid (Fig. 2b). No cystic cavities were observed at the cut (Fig. 2c). The gap in the joint capsule was covered using a bovine dura mater graft. Microscopic sections showed a variable cellularity: a central nodular area composed of dense fibrous connective tissue with focal areas of myxoid degeneration, and a peripheral dense fibrous connective tissue linked to the tendon sheath with some vascular structures (Fig. 3a-c). The histological diagnosis was of a fibroma of the tendon sheath. surrounded by abundant collagenic matrix. $\mathbf{c} H \& \mathrm{E} \times 40$, tumor cells appear to be fibroblasts with no evidence of atypical patterns

The patient was discharged the day after the operation with a physiotherapy program and an anterior splint to lock the joint in dorsal flexion, which was to be worn for two weeks. After one month he returned to his normal activities with full ROM of his ankle. This patient was followed up over a twelve-month period. In this time, he regained full function of his ankle without pain or recurrence of his previous symptoms. No swelling or recurrence of lesion was noted from the site of its excision.

The patient gave his consent to the publication of the clinical case.

\section{Discussion}

Fibroma of the tendon sheath, or tenosynovial fibroma, was first defined by Geschickter and Copeland in 1936 [5]. It has been described as a fibrotic neoplasm or a reactive 
fibrosis, but its precise origin is still unclear according to the current classification [3].

However, histologically it is clear that it is a poorly recognized, slowly growing, benign proliferation of fibroblasts surrounded by collagen fibers, which appears as a fibrous nodule attached to tendon or tendon sheath; a smooth, dense, multinodular mass with a diffuse pearly white appearance, ranging in size from 0.5 to $5.5 \mathrm{~cm}$. A dense, matrix-rich collagenous stroma is arranged in nodules with slit-like vascular channels throughout it. Occasionally myxoid and sclerotic regions are seen, depending probably on the vascular impairment due to compression. The cells are mainly spindle shaped and are less frequently stellate [2]. Hashimoto et al. [6] found that many of the cells are represented by myofibroblasts. Seventy-five to eighty-two percent of the tumors have been described in the extremities, most commonly the fingers, hands and wrists. The most important case report appears to be that of Chung and Enzinger [2] in 1979, who reported on 138 patients: $98 \%$ of cases occurred in those locations.

The tumor can occur at any age, with peak incidence occurring between 20 and 50 years. In the same paper, Chung and Enzinger reported a median age of 31. The male:female ratio has been described as 1.5-3:1 [2, 15].

The clinical presentation of tendon sheath fibroma often occurs years after its formation as a painless, slowly growing mass that may irritate the surrounding tissues by compression. Nerve compression has been described in the distal forearm, presenting itself as a median nerve neuropathy [1]. Less than $10 \%$ of patients have reported a history of trauma [15].

Diagnosis must be based on the patient history and clinical examination, MRI imaging and histology. Plain $\mathrm{X}$-rays are usually negative, except when large masses compress surrounding muscles or fat, or there are erosive bony changes, which are rarely described $[2,11]$. Various MRI findings have been reported: Bertolotto et al. [1] reported on a fibroma of the tendon sheath in the distal forearm and described a low MRI signal on T1-weighted images and a high signal on T2-wieghted images. Pinar et al. [14] reported on an intraarticular mass of the knee with the same MRI appearance. Hitora et al. [8] reported a low intensity in both T1- and T2-weighted images. Takakubo et al. [18] found a low intensity in T1-weighted images and a mixed low and high intensity in T2-weighted images. Fox et al. [4] described MRI findings in six cases, including a low intensity in T1-weighted images in five cases, low intensity and isointensity in T2-weighted images in three cases, and a slightly high intensity in T2-weighted images in two cases. The current interpretation of this kind of behavior is that differences in the amounts of hyalinization, sclerosis and the number of proliferating fibroblasts may generate variations in T2-weighted MRI findings: more hyalinized or sclerosed forms of FTS (fibroma of tendon sheath) will tend to show lower intensities on T2-weighted images, while a more cellular variant will have a higher T2 signal [17]. Gadolinium DTPAenhanced MRI variations have also been described: Pinar et al. [14] and Hitora et al. [8] described a diffuse contrast enhancement on Gd-DTPA-enhanced MRI; Takakubo et al. [18] described a peripheral enhancement which may be due to blood vessel proliferation at the periphery of the tumor.

Differential diagnosis must be made with giant cell tumor of the tendon sheath (GCTTS), representing a localized manifestation of pigmented villonodular synovitis that is less hyalinized and more cellular, and with histiocytes and monocytes as well as multinucleated giant cells, foam cells and hemosiderin-laden macrophages. Due to similarities between some forms of the two tumors, some authors have hypothesized that they may be two phenotypic extremities of a single entity [14]. FTS must also be distinguished from nodular fasciitis, which resembles FTS histologically but is a more rapidly growing mass $[2,6,15,16]$.

Treatment is by local excision, with a reported recurrence of 24\%; all of the cases were described in hands and fingers, and this probably depends on the accuracy of the excision itself [2]. To our knowledge, a malignant transformation has never been described.

In conclusion, we can affirm that this case is of particular interest due to the localization, with no clear continuity with any tendon or tendon sheath. To our knowledge, only seven cases of localization within a joint capsule have been described: four in the knee (two in the posterior joint capsule $[8,18]$, one arising from a posterior cruciate ligament [13], and one in the suprapatellar pouch [14]), one in the radioulnar joint [12], one in the temporomandibular joint [10], and one in the shoulder joint; rather unusually, this last one presented as multiple intraarticular loose bodies [7]. To our knowledge, ours is the first reported case of this tumor occurring in the ankle joint.

This case is reported to highlight the diagnosis of a FTS as a rare, but possible, cause of ankle joint mass.

Conflict of interest statement The authors declare that they have no conflict of interest related to the publication of this manuscript.

\section{References}

1. Bertolotto M, Rosemberg I, Parodi RC (1996) Case report: fibroma of tendon sheath in the distal forearm with associated median nerve neuropathy: US, CT and MR appearances. Clin Radiol 51:370-372

2. Chung EB, Enzinger FM (1979) Fibroma of tendon sheath. Cancer 44:1945-1954

3. Enzinger FM, Weiss WS (2001) Soft tissue tumors, 4th edn. Mosby, St Louis 
4. Fox MG, Kransdorf MJ, Bancroft LW (2003) MR imaging of fibroma of the tendon sheath. AJR 180:1449-1453

5. Geshickter CF, Copeland MM (1949) Tumors of the bone, 3rd edn. JB Lippincott, Philadelphia

6. Hashimoto H, Tsuneyoshi M, Daimaru Y, Ushijima M, Enjoji M (1985) Fibroma of tendon sheath: a tumor of myofibroblasts: a clinicopathologic study of 18 cases. Acta Pathol Jpn 35:1099-1107

7. Hermann G, Hoch BL, Springfield D, Abdelwahab IF, Klein MJ (2006) Intra-articular fibroma of tendon sheath of the shoulder joint: synovial fibroma. Skeletal Radiol 35(8):603-607

8. Hitora T, Yamamoto T, Akisue T, Marui T, Nagira K, Ohta R, Kurosaka M (2002) Fibroma of thendon sheath originating from the knee joint capsule. Clin Imaging 26(4):280-283

9. Hur J, Damron TA, Vermont AI, Mathur SC (1999) Fibroma of tendon sheath of the infrapatellar fat pad. Skeletal Radiol 28:407410

10. Li TJ, Kitano M, Tsuneyoshi M (1997) Intraarticular fibroma of tendon sheath in the temporomandibular joint. Oral Surg Oral Med Oral Pathol Oral Radiol Endod 84:407-410

11. Lourie JA, Lwin KY, Woods CG (1992) Case report 734, fibroma of tendon sheath eroding 3rd metatarsal bone. Skeletal Radiol 21:273-275
12. Misawa A, Okada K, Hirano Y, Sageshima M (1999) Fibroma of tendon sheath arising from the radio-ulnar joint. Pathol Int 49:1089-1092

13. Ogata K, Ushijima M (1987) Tenosynovial fibroma arising from the posterior cruciate ligament. Clin Orthop Relat Res 215:153155

14. Pinar H, Ozkan M, Ozaksoy D, Pabuccuoglu U, Akseki D, Karaoglan O (1995) Intraarticular fibroma of the tendon sheath of the knee. Arthroscopy 11:608-611

15. Pulitzer DR, Martin PC, Reed RJ (1989) Fibroma of tendon sheath: a clinicopathologic study of 32 cases. Am J Surg Pathol 13:472-479

16. Smith PS, Pieterse AS, McClure J (1982) Fibroma of tendon sheath. J Clin Pathol 35:842-848

17. Sundaram M, McGuire MH, Schajowicz F (1981) Soft tissue masses: histologic basis for decreased signal (short T2) on T2-weighted MR images. AJR 148:1241-1250

18. Takakubo Y, Fukushima S, Asano T, Yamakawa M (2005) Intraarticular fibroma of the tendon sheath in the knee. Clin Orthop 439:280-285 